\title{
Non-separable Banach spaces with non-meager Hamel basis
}

by

\author{
Taras Banakh (Kielce and Lviv), Mirna Džamonja (Norwich) and \\ Lorenz Halbeisen (Bern)
}

\begin{abstract}
We show that an infinite-dimensional complete linear space $X$ has:
- a dense hereditarily Baire Hamel basis if $|X| \leq \mathfrak{c}^{+}$;

- a dense non-meager Hamel basis if $|X|=\kappa^{\omega}=2^{\kappa}$ for some cardinal $\kappa$.
\end{abstract}

According to Corollary 3.4 of [BDHMP] each infinite-dimensional separable Banach space $X$ has a non-meager Hamel basis. This is a special case of Theorem 3.3 of [BDHMP], asserting that an infinite-dimensional Banach space $X$ has a non-meager Hamel basis provided $2^{d(X)}=d(X)^{\omega}$, where $d(X)$ is the density of $X$. Having in mind those results the authors of [BDHMP] asked if each infinite-dimensional Banach space has a non-meager Hamel basis. In this paper we shall give two partial answers to this question generalizing the abovementioned Corollary 3.4 and Theorem 3.3 of [BDHMP] in two directions.

THEOREM 1. Each infinite-dimensional linear complete metric space $X$ of size $|X| \leq \mathfrak{c}^{+}$has a dense hereditarily Baire Hamel basis.

We recall that a topological space $X$ is hereditarily Baire if each closed subspace $F$ of $X$ is Baire (in the sense that the intersection of a countable family of open dense subsets of $F$ is dense in $F$ ).

Our next result treats Banach spaces of even larger size. We define a subset $A$ of a topological space $X$ to be $\kappa$-perfect for some cardinal $\kappa$ if each non-empty open set $U$ of $A$ has size $|U| \geq \kappa$. Note that a Hausdorff space $X$ is $\omega$-perfect if and only if it has no isolated points (so is perfect in the standard sense).

It is well-known (see [BDHMP, 2.8]) that each Banach space $X$ has size $|X|=d(X)^{\omega}$. Our second principal result generalizes Theorem 3.3 of [BDHMP].

2000 Mathematics Subject Classification: 46B15, 03E75.

Key words and phrases: Hamel basis, Banach space, Baire space.

Mirna Džamonja thanks EPSRC for support through an Advanced Research Fellowship. 
THEOREM 2. If an infinite-dimensional linear complete linear space $X$ has size $|X|=\kappa^{\omega}=2^{\kappa}$ for some cardinal $\kappa$, then $X$ has a non-meager Hamel basis $H \subset X$ such that for any closed $|X|$-perfect subset $C \subset X$ the space $C \cap H$ is Baire.

Let us observe that there are many cardinals $\kappa$ with $\kappa^{\omega}=2^{\kappa}$.

Proposition 1. For any sequence of cardinals $\left(\kappa_{i}\right)_{i \in \omega}$ with $\kappa_{i+1} \geq 2^{\kappa_{i}}$, $i \in \omega$, the cardinal $\kappa=\sup _{i \in \omega} \kappa_{i}$ has the property $2^{\kappa}=\kappa^{\omega}$.

Proof. Since $\kappa^{\omega} \leq 2^{\kappa}$ always holds, it suffices to prove that $\kappa^{\omega} \geq 2^{\kappa}$. For this take a sequence $\left(X_{i}\right)_{i \in \omega}$ of pairwise disjoint sets of size $\left|X_{i}\right|=\kappa_{i}$ and let $X=\bigcup_{i \in \omega} X_{i}$. It is clear that $|X|=\kappa$ and the power set $\mathcal{P}(X)$ of $X$ has size $|\mathcal{P}(X)|=2^{\kappa}$. Since each subset $A=\bigcup_{i \in \omega} A \cap X_{i}$ of $X$ can be uniquely identified with the sequence $\left(A \cap X_{i}\right)_{i \in \omega}$, we get

$$
2^{\kappa}=|\mathcal{P}(X)|=\left|\prod_{i \in \omega} \mathcal{P}\left(X_{i}\right)\right|=\prod_{i \in \omega} 2^{\kappa_{i}} \leq \prod_{i \in \omega} \kappa_{i+1} \leq \kappa^{\omega} .
$$

In fact, one can make an easy observation about $\kappa^{\omega}$ which is helpful in calculating this value, and in particular implies Proposition 1. We use $\operatorname{cof}(\kappa)$ to denote the cofinality of $\kappa$.

Proposition 2. Suppose that $\operatorname{cof}(\kappa)=\aleph_{0}$. Then $2^{\kappa}=\left(\sup \left\{2^{\lambda}: \lambda<\kappa\right\}\right)^{\omega}$. If $\operatorname{cof}(\kappa)>\aleph_{0}$ then $\kappa^{\omega}=\kappa \cdot \sup \left\{\lambda^{\omega}: \lambda<\kappa\right\}$.

Proof. If $\kappa=\aleph_{0}$ then the proposed equality easily holds. Suppose that $\kappa>\aleph_{0}$. Then clearly $2^{\kappa}=\left(2^{\kappa}\right)^{\omega} \geq\left(\sup \left\{2^{\lambda}: \lambda<\kappa\right\}\right)^{\omega}$. Let $\left(\lambda_{i}\right)_{i \in \omega}$ be a sequence of regular cardinals increasing to $\kappa$, with $\lambda_{0}=0$, and let $\theta=$ $\sup \left\{2^{\lambda}: \lambda<\kappa\right\}$. Every subset $A$ of $\kappa$ can be identified with the sequence $\left(A \cap\left[\lambda_{i+1} \backslash \lambda_{i}\right)\right)_{i \in \omega}$, therefore $2^{\kappa} \leq\left|{ }^{\omega} \theta\right|=\theta^{\omega}$.

For the second equality, observe first that the left side of the equality is always no smaller than the right side. If $\operatorname{cof}(\kappa)>\aleph_{0}$ and $\kappa$ is a limit cardinal, then notice that every countable subset of $\kappa$ is already a subset of some $\lambda<\kappa$, so $\kappa^{\omega} \leq \sup \left\{\lambda^{\omega}: \lambda<\kappa\right\}$, which does not exceed the quantity on the right side of the equation. Finally, if $\kappa=\lambda^{+}$for some $\lambda$ then $\kappa^{\omega}=\bigcup_{\alpha \in[\lambda, \kappa)} \alpha^{\omega}$, and the latter set has size $\leq \kappa \cdot \lambda^{\omega} \leq 2^{\lambda}$, which is exactly the quantity on the right side of the equation.

Corollary 1. Suppose that a complete metric space $X$ satisfies $d(X) \in$ $\left[\kappa, 2^{\kappa}\right]$ for some $\kappa$ with $\kappa^{\omega}=2^{\kappa}$. Then $X$ contains a non-meager Hamel basis.

Under the Generalized Continuum Hypothesis GCH, each cardinal $\kappa$ of countable cofinality satisfies $\kappa^{\omega}=\kappa^{+}$. Consequently, each complete metric space $X$ with density $d(X) \in\left\{\kappa, \kappa^{+}\right\}$contains a non-meager Hamel basis.

Proof. Suppose that $d(X)=\lambda \in\left[\kappa, \kappa^{\omega}\right]$. Then $|X|=\lambda^{\omega}=\kappa^{\omega}=2^{\kappa}$, so $X$ contains a non-meager Hamel basis by Theorem 2 . For the conclusion 
under GCH notice that by König's lemma we have $\kappa^{\omega}>\kappa$, and since $\kappa^{\omega} \leq 2^{\kappa}$ we may conclude that $\kappa^{\omega}=\kappa^{+}$.

We comment that Corollary 1 shows that our Theorem 2 is more general then Theorem 3.3 of [BDHMP], since by assuming GCH and taking for example $X$ to be an infinite-dimensional Banach space of density $\lambda=\aleph_{\omega+1}$ (such as $\left.l_{\infty}\left(\aleph_{\omega}\right)\right)$, we find that $X$ has a non-meager Hamel basis by Corollary 1 , while $\lambda^{\omega}=\lambda<2^{\lambda}$ so Theorem 3.3 of [BDHMP] does not apply.

1. Proof of Theorem 1. The proof of Theorem 1 is divided into three lemmas. The first of them supplies us with many linearly independent Cantor sets.

A topological space $X$ is called a Cantor set if it is homeomorphic to the Cantor cube $\{0,1\}^{\omega}$. This happens if and only if $X$ is compact, metrizable, zero-dimensional and has no isolated points (see [Ke, 7.4]).

By the algebraic dimension of a subset $A$ of a linear space $L$ we understand the algebraic dimension (= the cardinality of a Hamel basis) of the linear hull $\operatorname{Lin}(A)$ of $A$ in $L$.

LEMMA 1. Let $L$ be a linear metric space and $L_{\infty}$ a linear subspace which can be written as the countable union $L_{\infty}=\bigcup_{n \in \omega} L_{n}$ of a non-decreasing sequence $\left(L_{n}\right)_{n \in \omega}$ of closed linear subspaces of $L$. Denote by $\pi: L \rightarrow L / L_{\infty}$ the quotient operator. Let $X \subset L$ be a completely metrizable subspace of $L$ such that for every non-empty open set $U \subset X$ the projection $\pi(U)$ has infinite algebraic dimension in $L / L_{\infty}$. Then $X$ contains a Cantor set $C \subset X$ whose projection is linearly independent in $L / L_{\infty}$ and has size $\mathfrak{c}$.

Proof. Fix a complete metric $\rho$ on $X$. Let $2=\{0,1\}$ and let $2^{<\omega}=$ $\bigcup_{n \in \omega} 2^{n}$ denote the set of finite binary sequences. For a binary sequence $s=\left(s_{1}, \ldots, s_{l}\right) \in 2^{<\omega}$ and $i \in\{0,1\}$, we denote by $\hat{s} i=\left(s_{1}, \ldots, s_{n}, i\right)$ the concatenation of $s$ and $i$.

By induction, to each sequence $s \in 2^{<\omega}$ we shall assign a non-empty open set $U_{s} \subset X$ so that the following conditions are satisfied for every $n \in \omega$ and $s \in 2^{n}$ :

(1) $\operatorname{diam}\left(U_{s}\right) \leq 2^{-n}$;

(2) $U_{s^{\wedge} 0} \cup U_{s^{\wedge} 1} \subset U_{s}$;

(3) $\bar{U}_{s \hat{} \wedge} \cap \bar{U}_{s^{\wedge} 1}=\emptyset$;

(4) for any points $x_{t} \in U_{t}, t \in 2^{n}$, and real numbers $\lambda_{t}, t \in 2^{n}$, the inclusion $\sum_{t \in 2^{n}} \lambda_{t} x_{t} \in L_{n}$ is possible only if all $\lambda_{t}=0$.

We put $U_{\emptyset}=X \backslash L_{0}$. Assume that for some $n$ the sets $U_{s}, s \in 2^{n}$, have been constructed. The projection $\pi(U)$ of each open set $U \subset X$ has infinite algebraic dimension. Consequently, for every finite-dimensional linear subspace $F$ of $L$ the intersection $\left(F+L_{n}\right) \cap U$ is nowhere dense in $U$. Using 
this fact, by finite induction of length $2^{n+1}$ in each set $U_{s}, s \in 2^{n}$, we can select two distinct points $x_{s^{\wedge} 0}, x_{s^{\wedge} 1} \in U_{s}$ so that the indexed set $\left\{x_{t}+\right.$ $\left.L_{n}: t \in 2^{n+1}\right\}$ is linearly independent in $L / L_{n}$. Next we can select open neighborhoods $U_{t}$ of the points $x_{t}$ to satisfy the conditions (1)-(4). This finishes the inductive construction.

Now it is easy to see that the intersection $C=\bigcap_{n \in \omega} \bigcup_{s \in 2^{n}} \bar{U}_{s}$ is a Cantor set in $X$. It follows from (4) that the image $\pi(C)$ in $L / L_{\infty}$ is linearly indepenedent and has size $\mathfrak{c}$.

Lemma 2. Let $L$ be a complete linear metric space of size $|L| \leq \mathfrak{c}$ and $\left(L_{n}\right)_{n \in \omega}$ be a non-decreasing sequence of closed linear subspaces of $L$ with infinite-dimensional quotient space $L / L_{\infty}$ where $L_{\infty}=\bigcup_{n \in \omega} L_{n}$. Let $H_{\infty}$ be a Hamel basis for $L_{\infty}$ such that for every $n \in \omega$ the intersection $H_{\infty} \cap L_{n}$ is a hereditarily Baire Hamel basis in $L_{n}$. Then $H_{\infty}$ can be enlarged to a dense hereditarily Baire Hamel basis $H$ for $L$.

Proof. Let $\pi: L \rightarrow L / L_{\infty}$ denote the quotient homomorphism and let $\mathcal{C}$ be the family of Cantor sets $C \subset L$ whose projection $\pi(C)$ on $L / L_{\infty}$ has algebraic dimension $\mathfrak{c}$. The family $\mathcal{C}$ has size $|\mathcal{C}| \leq|L|^{\omega} \leq \mathfrak{c}$ because each Cantor set $C \in \mathcal{C}$ is a continuous image of the Cantor cube $2^{\omega}$ and each continuous map $f: 2^{\omega} \rightarrow L$ is uniquely determined by values of $f$ on a countable dense subset of $2^{\omega}$. Let $\mathcal{C}=\left\{C_{\alpha}: \alpha<\mathfrak{c}\right\}$ be an enumeration of the family $\mathcal{C}$ by ordinals $<\mathfrak{c}$.

By transfinite induction we can construct a transfinite sequence of points $\left\{x_{\alpha}: \alpha<\mathfrak{c}\right\} \subset L$ so that $x_{\alpha} \in C_{\alpha} \backslash\left(L_{\infty}+\operatorname{Lin}\left\{x_{\beta}: \beta<\alpha\right\}\right)$. At each step $\alpha$ the choice of the point $x_{\alpha}$ is possible because each set $\pi\left(C_{\alpha}\right)$ has algebraic dimension $\mathfrak{c}$.

After completing the inductive construction we will get a set $E=\left\{x_{\alpha}\right.$ : $\alpha<\mathfrak{c}\}$ whose projection onto $L / L_{\infty}$ is injective and has linearly independent image in $L / L_{\infty}$. Then the union $H_{\infty} \cup E$ is a linearly independent subset of $L$ and can be enlarged to a Hamel basis $H$ for $L$. Since $H_{\infty}$ is a Hamel basis for $L_{\infty}$, we have $H \cap L_{\infty}=H_{\infty}$. We claim that the space $H$ is hereditarily Baire and dense in $L$.

To prove the density of $H$, take any non-empty open subset $U \subset L$. By Lemma 1, the set $U$ contains a Cantor set $C \subset U$ belonging to the family $\mathcal{C}$. By the inductive construction, $E \cap C \neq \emptyset$ and hence $H \cap U \neq \emptyset$ too.

Next we show that $H$ is hereditarily Baire. Assuming the converse and applying [De], we can find a closed countable subset $C \subset H$ without isolated points. Then the closure $\bar{C}$ of $C$ in $X$ is a Polish space without isolated points and so is the complement $\bar{C} \backslash C$. We claim that for each open set $U \subset \bar{C}$ the set $W=U \backslash H$ has an infinite-dimensional image $\pi(W)$ in $L / L_{\infty}$. The density of $\bar{C} \backslash H$ in $\bar{C}$ implies that $U \subset \bar{W}$. Assuming that $\pi(W)$ is finite-dimensional, we would find that $W \subset U \subset \bar{W} \subset L_{\infty}+F=\bigcup_{n \in \omega}\left(L_{n}+F\right)$ for some 
finite-dimensional linear subspace $F \subset L$ with $F \cap L_{\infty}=\{0\}$. The Baire theorem guarantees that some non-empty open subset of $U$ lies in $L_{n}+F$. Replacing $U$ by this open set we can assume that $U \subset L_{n}+F$. Since $H_{n}=$ $H \cap L_{n}=H_{\infty} \cap L_{n}$ is a Hamel basis for $L_{n}$, we have $H \cap\left(L_{n}+F\right)=H_{n} \cup B$ for some finite set $B$ disjoint from $L_{\infty}$. Then $U \cap H=U \cap\left(L_{n}+F\right) \cap H=$ $U \cap\left(H_{n} \cup B\right) \subset U \cap L_{n} \cup(U \cap B)$. We claim that $U \cap B=\emptyset$. Assuming the converse, we would infer that $U \cap B$ is a non-empty closed subset of $U \cap H$, which is not possible because $U \cap H=U \cap C$ has no isolated points. Thus $U \cap H=U \cap H_{n} \subset L_{n}$ is a countable set without isolated points in $H_{n}$, which contradicts the fact that $H_{n}=H \cap L_{n}$ is a hereditarily Baire Hamel basis for $L_{n}$.

Applying Lemma 2 to the sequence $\left(L_{n}\right)$ of trivial linear spaces $L_{n}=\{0\}$ we obtain a part of Theorem 1.

Lemma 3. Each infinite-dimensional linear complete metric space $X$ with $|X| \leq \mathfrak{c}$ contains a dense hereditarily Baire Hamel basis.

The remaining part of Theorem 1 is proved in

Lemma 4. Each complete metric linear space $X$ of size $|X|=\mathfrak{c}^{+}$contains a dense hereditarily Baire Hamel basis.

Proof. Given a complete linear metric space $X$ of size $|X|=\mathfrak{c}^{+}$, write $X$ as the union $X=\bigcup_{\alpha<\mathfrak{c}^{+}} X_{\alpha}$ of an increasing transfinite sequence $\left(X_{\alpha}\right)_{\alpha<\mathfrak{c}^{+}}$ of closed linear subspaces of size $\left|X_{\alpha}\right|=\mathfrak{c}$ such that, for every $\alpha<\mathfrak{c}^{+}$,

- the quotient $X_{\alpha+1} / X_{\alpha}$ is infinite-dimensional;

- $X_{\alpha}=X_{<\alpha}=\bigcup_{\beta<\alpha} X_{\beta}$ if $\alpha$ has uncountable cofinality;

- $X_{\alpha} / \bar{X}_{<\alpha}$ is infinite-dimensional if $\alpha$ has countable infinite cofinality.

It is convenient to assume that $X_{-1}=\{0\}$. By transfinite induction, for every $\alpha<\mathfrak{c}^{+}$we shall construct a dense hereditarily Baire Hamel basis $H_{\alpha}$ in $X_{\alpha}$ so that $H_{\alpha} \supset \bigcup_{\beta<\alpha} H_{\beta}$. To start the inductive construction let $H_{0}=\emptyset$.

Assume that for some ordinal $\alpha$, dense hereditarily Baire Hamel bases $H_{\beta}$ have been constructed in each space $X_{\beta}$ for $\beta<\alpha$. Now consider three cases:

1) $\alpha=\beta+1$ is a successor ordinal. In this case apply Lemma 2 with $L=X_{\alpha}$ and $L_{n}=X_{\beta}, n \in \omega$, to enlarge the Hamel basis $H_{\beta}$ to a dense hereditarily Baire Hamel basis $H_{\alpha}$ for the space $X_{\alpha}$.

2) $\alpha$ is a limit ordinal with countable cofinality. In this case we can find an increasing sequence of ordinals $\left(\alpha_{n}\right)_{n \in \omega}$ with $\alpha=\sup _{n} \alpha_{n}$ and apply Lemma 2 with $L=X_{\alpha}, L_{n}=X_{\alpha_{n}}$ and $H_{\infty}=\bigcup_{n \in \omega} H_{\alpha_{n}}$ to enlarge the Hamel basis $H_{\infty}$ to a dense hereditarily Baire Hamel basis $H_{\alpha}$ for $X_{\alpha}$.

3) $\alpha$ is of uncountable cofinality. In this case $X_{<\alpha}=\bigcup_{\beta} X_{\beta}$ and we can put $H_{\alpha}=\bigcup_{\beta<\alpha} H_{\beta}$. The density of the Hamel bases in $X_{\beta}$ implies the den- 
sity of $H_{\alpha}$ in $X_{\alpha}$. Let us show that the Hamel basis $H_{\alpha}$ is hereditarily Baire. Assuming the converse, and applying [De], we can find a closed countable subset $C \subset H_{\alpha}$ without isolated points. Since $\alpha$ has uncountable cofinality, $C \subset H_{\beta}$ for some $\beta<\alpha$. Then $H_{\beta}$ contains a closed meager subspace $C$ and thus is not hereditarily Baire, which is a contradiction.

2. Proof of Theorem 2. Given an infinite cardinal $\kappa$, we denote by $\sqrt[\omega]{\kappa}$ the smallest infinite cardinal $\lambda$ with $\lambda^{\omega} \geq \kappa$. The proof of Theorem 2 is similar to that of Theorem 1 and relies on

Lemma 5. For every $\kappa$-perfect complete metric space $X$ and a comeager subspace $G \subset X$ there is a subspace $\Pi \subset G$ homeomorphic to the countable product $\lambda^{\omega}$, where the cardinal $\lambda=\sqrt[\omega]{\kappa}$ is endowed with the discrete topology.

Proof. The complement $X \backslash G$, being meager in $X$, lies in the countable union $\bigcup_{n \in \omega} Z_{n}$ of closed nowhere dense subsets $Z_{n}$ in $X$. Since $X$ is $\kappa$-perfect, each non-empty open subset $U \subset X$ has size $|U| \geq \kappa$ and density $d(U) \geq \sqrt[\omega]{\kappa}=\lambda$. By the Erdôs-Tarski theorem [ET] (see also [En, 4.1.H]), the metrizable space $X \backslash Z_{0}$ contains a family $\mathcal{U}_{0}$ consisting of $\lambda$ many open subsets of $X \backslash Z_{0}$ of diameter $<1 / 2^{0}$ such that the family $\overline{\mathcal{U}}_{0}=\{\bar{U}$ : $\left.U \in \mathcal{U}_{0}\right\}$ is disjoint. Repeating this argument, inductively construct a sequence $\left(\mathcal{U}_{n}\right)_{n \in \omega}$ of families of non-empty open sets of $X \backslash Z_{n}$ having diameter $<1 / 2^{n}$ so that $\overline{\mathcal{U}}=\left\{\bar{U}: U \in \mathcal{U}_{n}\right\}$ is disjoint, $\bigcup \overline{\mathcal{U}}_{n+1} \subset \bigcup \mathcal{U}_{n}$ and for every $U \in \mathcal{U}_{n}$ the family $\mathcal{U}_{n+1}(U)=\left\{W \in \mathcal{U}_{n+1}: \bar{W} \subset U\right\}$ has size $\lambda$. It is easy to see that the space $F=\bigcap_{n \in \omega} \bigcup \overline{\mathcal{U}}_{n} \subset X \backslash \bigcup_{n \in \omega} Z_{n} \subset G$ is homeomorphic to the product $\prod_{n \in \omega} \mathcal{U}_{n}$ where each $\mathcal{U}_{n}$ is endowed with the discrete topology, and the latter product is homeomorphic to $\lambda^{\omega}$.

With Lemma 5 in hand, we are now able to present

Proof of Theorem 2. Let $X$ be an infinite-dimensional linear complete metric space of size $|X|=2^{\kappa}=\kappa^{\omega}$ for some cardinal $\kappa$. Without loss of generality, $\kappa$ is the smallest infinite cardinal with that property. If $|X| \leq \mathfrak{c}$, then $X$ has a hereditarily Baire Hamel basis by Theorem 1 and we are done. So asume that $|X|>\mathfrak{c}$ and hence $\kappa>\omega$.

Let $\mathcal{K}$ denote the family of all subspaces $K \subset X$ that are homeomorphic to the countable product $\kappa^{\omega}$ where $\kappa$ is endowed with the discrete topology. Observe that each embedding $f: \kappa^{\omega} \rightarrow X$ is uniquely determined by the values of $f$ on a dense subset of $\kappa^{\omega}$. Since $\kappa^{\omega}$ has density $\kappa$, the family $\mathcal{K}$ has size $|\mathcal{K}| \leq|X|^{\kappa}=\left(2^{\kappa}\right)^{\kappa}=2^{\kappa}=|X|$ and hence can be enumerated as $\mathcal{K}=\left\{K_{\alpha}: \alpha<|X|\right\}$. Observe that each space $K \in \mathcal{K}$ has size $|K|=\kappa^{\omega}>\mathfrak{c}$ and algebraic dimension $\kappa^{\omega}$.

By transfinite induction we can construct a transfinite sequence of points $\left\{x_{\alpha}: \alpha<\mathfrak{c}\right\} \subset X$ so that $x_{\alpha} \in K_{\alpha} \backslash \operatorname{Lin}\left\{x_{\beta}: \beta<\alpha\right\}$. At each step $\alpha$ the 
choice of the point $x_{\alpha}$ is possible because each set $K_{\alpha}$ has algebraic dimension $\kappa^{\omega}>\alpha$.

After completing the inductive construction we will get a linearly independent set $E=\left\{x_{\alpha}: \alpha<\mathfrak{c}\right\}$ that meets each set $K \in \mathcal{K}$. Complete $E$ to a Hamel basis $H \supset E$.

We claim that for each closed $|X|$-perfect subset $F \subset X$ the intersection $F \cap H$ is non-meager. Assuming the converse, we can apply Lemma 5 to find a topological copy $K \subset F \backslash H$ of $\kappa^{\omega}$. It follows from the construction of $H$ that $K \cap H \neq \emptyset$, which contradicts the inclusion $K \subset F \backslash H$.

3. Some remarks and open problems. Our Theorem 2 generalizes Corollary 3.4 of [BDHMP] supplying a non-meager Hamel basis in each Banach space $X$ whose density $d(X)$ satisfies the equality $2^{d(X)}=d(X)^{\omega}$. In its turn, this corollary was derived from Theorem 3.3 of [BDHMP] guaranteeing the existence of a non-meager Hamel basis in each Banach space $X$ satisfying $\operatorname{cof}\left(\mathcal{M}_{X}\right) \leq|X|$, where $\operatorname{cof}\left(\mathcal{M}_{X}\right)$ stands for the cofinality of the ideal of meager sets in $X$. Having this result in mind, the authors of [BDHMP] asked in [BDHMP, Question 2] if the inequality $\operatorname{cof}\left(\mathcal{M}_{X}\right)>|X|$ holds for a suitable Banach space $X$. This is indeed so if $d(X)=|X|$. We shall prove a somewhat more general result giving lower and upper bounds for the cardinal $\operatorname{cof}\left(\mathcal{M}_{X}\right)$ via the weight $w(X)$ and the cellularity $c(X)$ of a linear topological space $X$.

Proposition 3. Let $X$ be a Baire topological space without isolated points. Then

(1) $\operatorname{cof}\left(\mathcal{M}_{X}\right) \leq w(X)^{c(X)}$;

(2) $\operatorname{cof}\left(\mathcal{M}_{X}\right)>|\mathcal{U}|$ for any disjoint family $\mathcal{U}$ of open sets in $X$.

Proof. (1) Fix a base $\mathcal{B}$ of the topology of $X$ of size $|\mathcal{B}|=w(X)$. Let $\mathcal{N}=\{X \backslash \bigcup \mathcal{U}: \mathcal{U} \subset \mathcal{B},|\mathcal{U}| \leq c(X)\}$. It is clear that $|\mathcal{N}| \leq w(X)^{c(X)}$. We claim that each nowhere dense subset $Z \subset X$ lies in some set $N \in \mathcal{N}$. Indeed, take a maximal disjoint subfamily $\mathcal{U} \subset \mathcal{B}$ with $\bigcup \mathcal{U} \subset X \backslash Z$ and note that $|\mathcal{U}| \leq c(X)$. Then $Z \subset X \backslash \cup \mathcal{U} \in \mathcal{N}$. It follows that the family $\mathcal{N}_{\infty}=\{\bigcup \mathcal{C}: \mathcal{C}$ is a countable subfamily of $\mathcal{N}\}$ is cofinal in $\mathcal{M}_{X}$ and has size $\left|\mathcal{N}_{\infty}\right| \leq|\mathcal{N}|^{\omega} \leq\left(w(X)^{c(X)}\right)^{\omega}=w(X)^{c(X)}$. Then $\operatorname{cof}\left(\mathcal{M}_{X}\right) \leq\left|\mathcal{N}_{\infty}\right| \leq$ $w(X)^{c(X)}$.

(2) Assume conversely that $\operatorname{cof}\left(\mathcal{M}_{X}\right) \leq|\mathcal{U}|$ for some disjoint family $\mathcal{U}$ of non-empty open sets in $X$. Pick a cofinal family $\mathcal{M}$ in $\mathcal{M}_{X}$ of size $|\mathcal{M}| \leq|\mathcal{U}|$ and enumerate $\mathcal{M}=\left\{M_{U}: U \in \mathcal{U}\right\}$ by elements of the family $\mathcal{U}$. Each open set $U \in \mathcal{U}$ is not meager because $X$ is Baire. Consequently, $U \not \subset M_{U}$ and we can pick a point $x_{U} \in U \backslash M_{U}$. Then the set $A=\left\{x_{U}: U \in \mathcal{U}\right\}$, being discrete, is nowhere dense in $X$. On the other hand, $A$ lies in no set $M \in \mathcal{M}$, which means that $\mathcal{M}$ is not cofinal in the ideal $\mathcal{M}_{X}$. 
Since each metrizable space $X$ contains a disjont family $\mathcal{U}$ of open sets of size $|\mathcal{U}|=d(X)$ (see $[\mathrm{ET}]$ or [En, 4.1.H]), Proposition 3 implies the following corollary answering Question 2 of [BDHMP].

Corollary 2. For any metrizable Baire space $X$ without isolated points we get $d(X)<\operatorname{cof}\left(\mathcal{M}_{X}\right) \leq 2^{d(X)}$.

A typical linear topological space with countable cellularity is the Tikhonov product $\mathbb{R}^{\kappa}$ of $\kappa$ many lines. Then repeating the argument of the proof of Theorem 3.3 [BDHMP] we can prove

Proposition 4. For any infinite cardinal $\kappa$ the linear topological space $X=\mathbb{R}^{\kappa}$ has a non-meager Hamel basis and satisfies $\operatorname{cof}\left(\mathcal{M}_{X}\right) \leq \kappa^{\omega} \leq 2^{\kappa}=|X|$.

In spite of (partial) results proven in this paper we still do not know the complete answer to the basic

Problem 1. Let $X$ be an infinite-dimensional Banach space.

(1) Does $X$ have a non-meager Hamel basis?

(2) Does $X$ have a non-meager Hamel basis if $|X|=\mathfrak{c}^{++}$?

(3) Does $X$ have a Hamel basis containing no uncountable compact subset?

\section{References}

[BDHMP] T. Bartoszyński, M. Džamonja, L. Halbeisen, E. Murtinová and A. Plichko, On bases in Banach spaces, Studia Math. 170 (2005), 147-171.

[De] G. Debs, Espaces héréditairement de Baire, Fund. Math. 129 (1988), 199-206.

[En] R. Engelking, General Topology, PWN, Warszawa, 1977.

[ET] P. Erdős and A. Tarski, On families of mutually exclusive sets, Ann. of Math. 44 (1943), 315-329.

[Ke] A. Kechris, Classical Descriptive Set Theory, Springer, New York, 1995.

Instytut Matematyki

Uniwersytet Humanistyczno-Przyrodniczy

im. Jana Kochanowskiego w Kielcach

Świętokrzyska 15

25-406 Kielce, Poland

and

Department of Mathematics

Ivan Franko National University of Lviv

1 Universytetska St.

79000 Lviv, Ukraine

E-mail: tbanakh@yahoo.com
Department of Mathematics University of East Anglia Norwich, NR4 7TJ, United Kingdom E-mail: M.Dzamonja@uea.ac.uk Theoretische Informatik und Logik Universität Bern Neubrückstr. 10 3012 Bern, Switzerland E-mail: halbeis@iam.unibe.ch 